\title{
Localized Neuronal Outgrowth Induced by Long-Term Sensitization Training in Aplysia
}

\author{
Marcy L. Wainwright, Han Zhang, John H. Byrne, and Leonard J. Cleary \\ W. M. Keck Center for the Neurobiology of Learning and Memory, Department of Neurobiology and Anatomy, University \\ of Texas-Houston Medical School, Houston, Texas 77030
}

Biophysical, biochemical, and morphological studies have implicated sensory neurons as key sites of plasticity in the formation and retention of the memory of long-term sensitization in Aplysia californica. This study examined the effects of different sensitization training protocols on the structure of sensory neurons mediating the tail-siphon withdrawal reflex. A $4 \mathrm{~d}$ training period produced a robust localized outgrowth in these sensory neurons observed $24 \mathrm{hr}$ after the end of training. These changes are consistent with previous results in siphon sensory neurons (Bailey and Chen, 1988a). In contrast, $1 \mathrm{~d}$ of sensitization training, which has been shown to effectively induce long-term behavioral sensitization and synaptic facilitation (Frost et al., 1985; Cleary et al., 1998), is not associated with morphological changes in tail sensory neurons at either $24 \mathrm{hr}$ or $4 \mathrm{~d}$ after training. Similarly, a single treatment with the growth factor TGF- $\beta$, which also induced facilitation, did not alter sensory neuron morphology. The different effectiveness of the two protocols was not simply a reflection of the number of stimuli presented, because a $1 \mathrm{~d}$ massed training protocol did not produce sensitization $24 \mathrm{hr}$ after training, nor did it induce neuronal outgrowth.

These results suggest that extensive sensitization training is required to induce neuronal outgrowth in tail sensory neurons, indicating that the memory of long-term sensitization induced by $1 \mathrm{~d}$ of training is mechanistically different from that induced by $4 \mathrm{~d}$ of training. Moreover, the induction of a form of long-term sensitization associated with neuronal outgrowth does not appear to be a function of the amount of stimulation but does appear to be dependent on the temporal spacing of the stimulation over multiple days.

Key words: morphology; Aplysia; sensitization; long-term memory; nonassociative learning; neuronal outgrowth
The possibility that morphological changes play a role in learning and memory has intrigued neuroscientists for many years. Ramón y Cajal hypothesized more than a century ago that the structure of individual neurons was plastic and modifiable by experience throughout life (Ramón y Cajal, 1988). Over the last few decades, empirical evidence has been found to support this hypothesis in a number of systems (for reviews, see Greenough and Bailey, 1988; Bailey and Kandel, 1993; Rosenzweig, 1996). The number and variety of examples in which modified neuronal structure is correlated with learning and memory suggest that morphological changes may play an important role in neuronal plasticity.

A good model system for studying cellular and molecular plasticity is the defensive withdrawal reflex of the marine mollusk Aplysia. Two such reflexes, the tail-siphon withdrawal reflex (Walters et al., 1983b; Scholz and Byrne, 1987) and the siphon-gill withdrawal reflex (Byrne et al., 1974; Frost et al., 1985), have been studied extensively and have contributed greatly to understanding the mechanisms of learning and memory. Both reflexes can be enhanced by sensitization, a simple form of nonassociative learning, in which the behavioral response to a mild stimulus is enhanced after presentation of a noxious stimulus (Castellucci et al., 1970; Carew et al., 1971; Walters et al., 1983b; Cleary et al.,

Received Dec. 17, 2001; revised Feb. 6, 2002; accepted Feb. 13, 2002.

This work was supported by National Institutes of Health Grants T32 NS07373 (M.L.W.), F31 MH12176 (M.L.W.), R01 NS019895 (J.H.B.), and R01 NS038100 (L.J.C.). We thank Kara Herynk and Jennifer Foxx for technical assistance and many hours of animal testing.

Correspondence should be addressed to Leonard J. Cleary, Department of Neurobiology and Anatomy, University of Texas-Houston Medical School, 6431 Fannin Street, Houston, TX 77030. E-mail: len.cleary@uth.tmc.edu.

Copyright (C) 2002 Society for Neuroscience $0270-6474 / 02 / 224132-10 \$ 15.00 / 0$
1998). In Aplysia, the effects of sensitization are specific to the area of training (Scholz and Byrne, 1987; Walters, 1987). Although sensitization is commonly viewed as a short-term process, lasting only minutes to hours (Dudai, 1989; Domjan, 1993), repeated presentation of noxious stimuli in Aplysia leads to a long-term form lasting from $1 \mathrm{~d}$ to $>3$ weeks depending on the stimulation protocol (Pinsker et al., 1973; Frost et al., 1985; Scholz and Byrne, 1987; Bailey and Chen, 1989). Short-term and long-term sensitization appear to share common features but are distinguishable in two ways. First, although the short-term change involves modification of preexisting proteins and is unaffected by inhibitors of transcription and translation, the induction of longterm changes is sensitive to these inhibitors (Montarolo et al., 1986; Castellucci et al., 1989; Levenson et al., 1999).

Second, whereas short-term sensitization appears to involve the strengthening of preexisting connections, long-term sensitization has been associated with neuronal outgrowth (Bailey and Chen, 1988a, 1989). In the siphon-gill withdrawal reflex, long-term sensitization leads to outgrowth of processes and formation of new varicosities in the sensory neurons that comprise the afferent limb of the reflex (Bailey and Chen, 1983, 1988a,b, 1989). This outgrowth would presumably result in the formation of new synapses onto the motor neurons controlling the movement of the siphon. Transcription- and translation-dependent structural changes are also induced by in vitro analogs of long-term sensitization training (Montarolo et al., 1986; Glanzman et al., 1990; Nazif et al., 1991; O'Leary et al., 1995; Casadio et al., 1999). Consequently, morphological changes are thought to be a general correlate of long-term sensitization in Aplysia.

Tail sensory neurons in the pleural ganglion appear to be the 
key site of plasticity in the tail-siphon withdrawal reflex. Tail sensory neurons synapse directly onto the tail motor neurons in the pedal ganglion (Walters et al., 1983) (see Fig. 2A). They also make polysynaptic connections onto motor neurons in the abdominal ganglion that control the withdrawal of the siphon (Perlman, 1979; Cleary and Byrne, 1993; Cleary et al., 1995). Sensitization training leads to several biophysical modifications in the tail sensory neurons, including increased excitability, enhanced synaptic strength, and a reduction of net outward currents (Scholz and Byrne, 1987; Walters, 1987; Cleary et al., 1998). In vitro analogs of sensitization training have demonstrated that the pleural sensory neurons also undergo structural plasticity (Glanzman et al., 1990; Nazif et al., 1991; Schacher et al., 1993), but the structure of these cells after sensitization training has not been examined in vivo. In addition, morphological correlates have been observed only after $4 \mathrm{~d}$ of training. Long-term sensitization lasting at least $24 \mathrm{hr}$ can be induced by a single day of training, but it is not known whether this sensitization is also correlated with morphological changes.

In this study, we examined the effect of sensitization training in freely behaving animals on the neuronal outgrowth in tail sensory neurons. Specifically, we compared the effects of $4 \mathrm{~d}$ and $1 \mathrm{~d}$ training protocols on large-scale morphological changes that might reflect the formation of new synapses, such as the extent and complexity of the neuritic processes and number of presynaptic varicosities. The results suggest that such changes are correlated with some, but not all, forms of long-term sensitization. Moreover, several observations cast doubt on a causal role for neurite outgrowth (and new synapse formation) in long-term sensitization.

\section{MATERIALS AND METHODS}

\section{Behavioral testing and training}

Aplysia californica (100-300 gm) were obtained from Alacrity Marine Biological (Redondo Beach, CA) and Marinus Inc. (Long Beach, CA). Animals were housed in individual cages in $15^{\circ} \mathrm{C}$ artificial seawater (Instant Ocean, Aquarium Systems, Mentor, $\mathrm{OH}$ ), exposed to a $12 \mathrm{hr}$ light/dark cycle, and fed sufficient dried seaweed to maintain constant body weight. Aplysia were allowed to recover from shipping for at least $3 \mathrm{~d}$ before any manipulation (Levenson et al., 1999). At least 1 week before behavioral testing, the parapodia were surgically trimmed to improve visualization of the siphon. Because food intake might have short-lasting inhibitory effects on the siphon withdrawal response (Advokat, 1980), animals were not fed for at least $24 \mathrm{hr}$ before the beginning of the experiment and were food deprived throughout (Levenson et al., 1999). Conditions were adjusted so that animals were without food for equal amounts of time preceding the post-test.

Behavioral testing and training were performed as reported previously (Scholz and Byrne, 1987; Goldsmith and Byrne, 1993) (Fig. 1). Briefly, the tail-siphon withdrawal response was elicited by mild AC electrical stimulation (20 msec duration) administered through two pairs of Teflon-coated silver wire electrodes (Ag 5T, Medwire) implanted in either side of the tail. The specific location depended on animal size, but in general electrodes were placed $\sim 1 \mathrm{~cm}$ from the tip of the tail and 0.5 $\mathrm{cm}$ from the midline (Scholz and Byrne, 1987; Cleary et al., 1998). Electrode placement was confirmed at the end of the experiment by dissection of the tail. Animals were excluded from the study if any of the exposed silver wire was visible outside of the tail, if exposed wires were in contact inside the tail, or if an electrode crossed over the midline.

The intensity of the stimulus used to elicit siphon withdrawal varied among animals and was determined by the threshold intensity, which is defined as the minimum intensity of stimulation that elicits a response. Thirty minutes before the start of the experiment, threshold was determined for both sides of the animal by applying $20 \mathrm{msec}$ shocks to the tail in $0.2 \mathrm{~mA}$ intensity intervals until a detectable siphon withdrawal was elicited. The intensity of the test stimulus was set at twice the threshold.

To establish a baseline for siphon withdrawal duration, 10 test stimuli were applied at $5 \mathrm{~min}$ intervals to alternate sides of the tail $(5$ on each
A.



B. 1-day Training Protocol



Sensitizing $\longrightarrow, \frac{10^{\prime \prime}}{30^{\prime}}$
Stimuli

C. 4-day Training Protocol

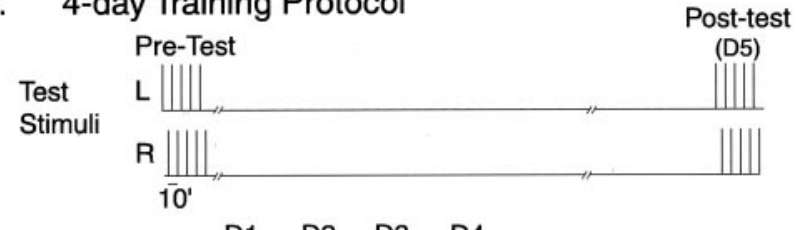

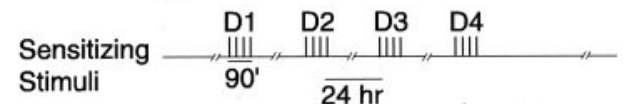

D. Massed Training Protocol

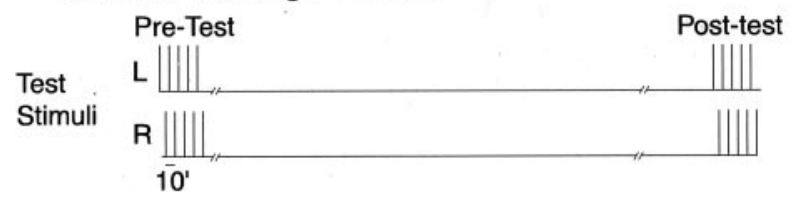



Figure 1. Testing and training protocols. A, Dorsal view of Aplysia. Mild electrical stimuli, administered through two pairs of electrodes implanted in either side of the tail (Test Site), were used to elicit the siphonwithdrawal response. Sensitizing stimuli were distributed along the lateral body wall (hatched area) of one randomly chosen side of the animal via hand-held electrodes. $B$, One day training protocol. Pre-test stimuli were applied at $5 \mathrm{~min}$ intervals to alternating sides of the tail immediately before training. There were $10 \mathrm{~min}$ between successive shocks to the left $(L)$ and right $(R)$ sides. For the periods before and after training, each vertical line represents one $20 \mathrm{msec}$ stimulation to the tail. Sensitization training commenced $5 \mathrm{~min}$ after the last pretest stimulus and consisted of a block of four separate trains of 10 shocks, separated by $30 \mathrm{~min}$. The duration of the siphon withdrawal response was measured again $24 \mathrm{hr}$ after the end of the last training session (Post-test) to determine the magnitude of sensitization produced by training. For the sensitizing stimuli, each vertical line represents a $60 \mathrm{~mA}, 500 \mathrm{msec}$ shock $(500 \mathrm{msec}$ interstimulus interval) to the body wall. $C$, Four day training protocol. The $1 \mathrm{~d}$ protocol was repeated for 4 consecutive days $(D 1-D 4)$ at $24 \mathrm{hr}$ intervals for the $4 \mathrm{~d}$ training protocol. The post-test was performed $24 \mathrm{hr}$ after the last day of training, on day 5 (D5). For the sensitizing stimuli, each vertical line represents one $90 \mathrm{~min}$ training session (four $10 \mathrm{sec}$ trains separated by $30 \mathrm{~min}$, as detailed in $B$ ). $D$, Massed training protocol. For the massed training protocol, animals were administered 16 trains of stimuli with trains separated by $30 \mathrm{~min}$. The training session lasted $7.5 \mathrm{hr}$. Post-tests were administered $22 \mathrm{hr}$ after the end of training. For the sensitizing stimuli, each vertical line represents one sensitizing stimulus. 
side) immediately before training (pre-test). Siphon withdrawal was measured as the time between initiation of withdrawal and reversal of motion (relaxation) of the siphon. Test stimuli also elicited tail withdrawal, but this response was difficult to assess in freely moving animals and was not included in these studies. Animals were excluded from the study if they inked or secreted opaline before training or had average withdrawal durations $>10 \mathrm{sec}$ in the pre-test, because these conditions indicated unhealthy or already sensitized animals.

Sensitization training commenced 5 min after the last pre-test stimulus. The stimulus used for sensitization training was a strong AC electric shock ( $60 \mathrm{~mA}, 500 \mathrm{msec}$ duration) delivered through a hand-held electrode. In all experiments, trains of 10 stimuli $(1 \mathrm{~Hz})$ were applied diff usely to the lateral body wall of one randomly chosen side of the animal via a handheld electrode (Fig. $1 A$, hatched area). The left side was used for training in roughly the same number of experiments as the right. This permitted comparison of the behavioral modification on the side of the animal ipsilateral to the training site with that on the contralateral side without biasing toward a particular side. The stimuli were applied to an area outside of known receptive fields of tail sensory neurons (Walters et al., 1983a). These stimuli reliably induced inking and/or opaline secretion. Previous studies (Scholz and Byrne, 1987; Cleary et al., 1998) have shown that sensitization training leads to a unilateral enhancement of the tail-induced siphon withdrawal reflex on the trained side. Untrained animals were treated identically to their trained counterparts but did not receive the sensitizing stimuli.

The timing and patterning of trains was determined by the specific protocol. In general, a single session consisted of four trains of sensitizing stimuli presented at intervals of $30 \mathrm{~min}$. The $1 \mathrm{~d}$ protocol (Fig. 1B) consisted of one such session (Scholz and Byrne, 1987; Goldsmith and Byrne, 1993; Cleary et al., 1998). In one experiment, the intertrain interval was $12 \mathrm{~min}$. The $4 \mathrm{~d}$ protocol (Fig. 1C) consisted of one session repeated over 4 successive days, with each session separated by $24 \mathrm{hr}$. In a third protocol (Fig. $1 D$ ), the same number of trains delivered in the $4 \mathrm{~d}$ protocol were delivered in a single day. The intertrain interval was kept at $30 \mathrm{~min}$, making the total duration of the training session $7.5 \mathrm{hr}$.

In general, the duration of the siphon withdrawal was measured again $24 \mathrm{hr}$ after the end of the last training block (post-test) to determine the magnitude of sensitization produced by the training. For the massed training protocol (Fig. $1 D$ ), the post-test occurred $22 \mathrm{hr}$ after training. The same stimulus intensity was used for both pre-tests and post-tests.

In all experiments, different investigators performed testing and training, and the tester was unaware of the previous treatment of the animals. All behavioral experiments were conducted at $15^{\circ} \mathrm{C}$.

\section{Sensory neuron reconstructions}

Immediately after the post-test, animals were anesthetized by injection of isotonic $\mathrm{MgCl}_{2}(500 \mathrm{ml} / \mathrm{kg})$, and both pleural-pedal ganglia were removed. Tail sensory neurons were identified by size, by location within the ventrocaudal sensory neuron cluster, and by the antidromic action potential resulting from stimulation of the peripheral nerve P9 (Walters et al., 1983a). Dextran-conjugated tetramethylrhodamine (Rh-dextran; 3000 MW; Molecular Probes, Eugene, OR; in $0.9 \% \mathrm{KCl}$ ) was injected by pressure into a single tail sensory neuron in each pleural ganglion until the cell body was visibly red in color. Ganglia were then placed in a $15^{\circ} \mathrm{C}$ incubator for $4 \mathrm{hr}$ to allow diffusion of the dye throughout the neuronal arborization. After incubation, ganglia were fixed in paraformaldehyde ( $4 \%$ in PBS $+30 \%$ sucrose) and cryosectioned at a nominal thickness of $40 \mu \mathrm{m}$. Slides were mounted in an antifade medium (Prolong, Molecular Probes) to minimize photobleaching during reconstruction.

Sensory neurons from the ganglion on the side of the animal that received sensitization training are referred to as ipsilateral cells. Those taken from the opposite side of the trained animal are referred to as contralateral cells. Before sectioning, ganglia were coded so that the investigator reconstructing the cells was unaware of the training conditions.

Three-dimensional structures of the labeled tail sensory neurons were quantified using a Neurolucida system (Microbrightfield, Inc., Baltimore, MD) interfaced to an upright microscope (Zeiss Axioscope) with a $40 \times$ (1.0 numerical aperture) water-immersion objective. This system tracks the $x, y$, and $z$ coordinates allowing a three-dimensional reconstruction of the cell to be produced. The sensory neuron was followed through serial sections to capture its structure throughout the pleural and pedal ganglia (Fig. 2). Four measures were used to characterize the morphology of each sensory neuron: total arborization length (in micrometers), number of branch points, number of first-order branches, and number of varicos- ities. Arborization length refers to the summed length of all processes (excluding the main axon). Branch points refer to the number of nodes at which the arborization bifurcates (trif urcating processes were treated as two bifurcating processes). Branches extending directly off the main axon were called first-order branches. Varicosities were defined as neuritic swellings that appeared to be at least $1.5 \times$ the diameter of the surrounding axon (Bailey et al., 1979). There are several types of varicosities, including small bead-like swellings along branches, swellings at branch points, and terminal varicosities that vary in size and shape (Bailey and Chen, 1988a). For the purposes of this study, no attempt was made to distinguish among the different types. To assure sufficient filling of all neurons, cells were included in the study only if the labeling remained bright throughout the pedal ganglion and into the peripheral nerve, and if there was no evidence of damage to the cell.

\section{Statistical analysis}

Behavioral measurements. For each animal, the averages of the five test scores (siphon withdrawal duration, in seconds) before and after training were calculated, and the percentage change in the average test score after training relative to the average score before training was calculated for each side (ipsilateral and contralateral). For the untrained control group, scores from the left and right sides were randomly assigned into two groups to mimic the random assignments in the trained animals. The values between these two untrained groups did not differ significantly for any of the experiments and were pooled for analysis. Behavioral scores were averaged for each group and compared using a one-way ANOVA. The Tukey test was used for post hoc multiple comparisons.

Morphological correlates. Because there were no differences between the cells from the ipsilateral and contralateral ganglia in trained animals (indicating that changes were dependent on whether the animal was trained regardless of which side received the training), we grouped the data into "trained" and "untrained." The trained group consisted of cells from ipsilateral and contralateral sides of trained animals, and the untrained group consisted of cells from both the left and right sides of untrained animals. For each morphological parameter measured (arborization length, first-order branches, branch points, and varicosities), the data were averaged for each group, and the groups were compared using Student's two-tailed unpaired $t$ test.

Because the four parameters were measured from the same populations of cells, it is important to confirm that these parameters are independent. In untrained animals from the $4 \mathrm{~d}$ training experiment, a correlation analysis of the six different parameter pairs revealed only one that showed a significant correlation (arborization length and number of branch points; $\left.r^{2}=0.87 ; p<0.005 ; n=7\right)$. Although highly correlated in control animals, this pair of parameters is indeed independent. In a separate study (Wainwright et al., 1999), cells that were damaged by axonal crush responded with a significant increase in total arborization length without a change in the number of branch points. Conversely, undamaged cells from the ganglion contralateral to the injury site responded with a significant increase in the number of branch points without a change in arborization length.

\section{RESULTS}

\section{Four days of training induced outgrowth in the pedal arborization}

Four days of sensitization training produced a robust unilateral enhancement of the reflex $24 \mathrm{hr}$ after training $\left(F_{(2,37)}=36.36\right.$; $p<0.0001$ ) (Fig. 3A). That is, the duration of the siphon withdrawal response elicited by mild tail stimulation on the side of the animal that received sensitization training was significantly enhanced compared with the response before training. Post hoc comparisons revealed that siphon withdrawal duration was significantly greater on the side ipsilateral to the training site compared with both the contralateral side (438 \pm 49 vs $138 \pm 24 \%$; mean $\pm \mathrm{SEM} ; q=9.49 ; p<0.001$; Tukey) and with untrained control animals (438 \pm 49 vs $107 \pm 16 \%$; $q=11.47 ; p<0.001$; Tukey).

Immediately after the behavioral post-test $(24 \mathrm{hr}$ after the last day of training), sensory neurons were prepared for morphological analysis. Quantitative analysis of sensory neuron structure revealed only modest changes. There were no differences between 


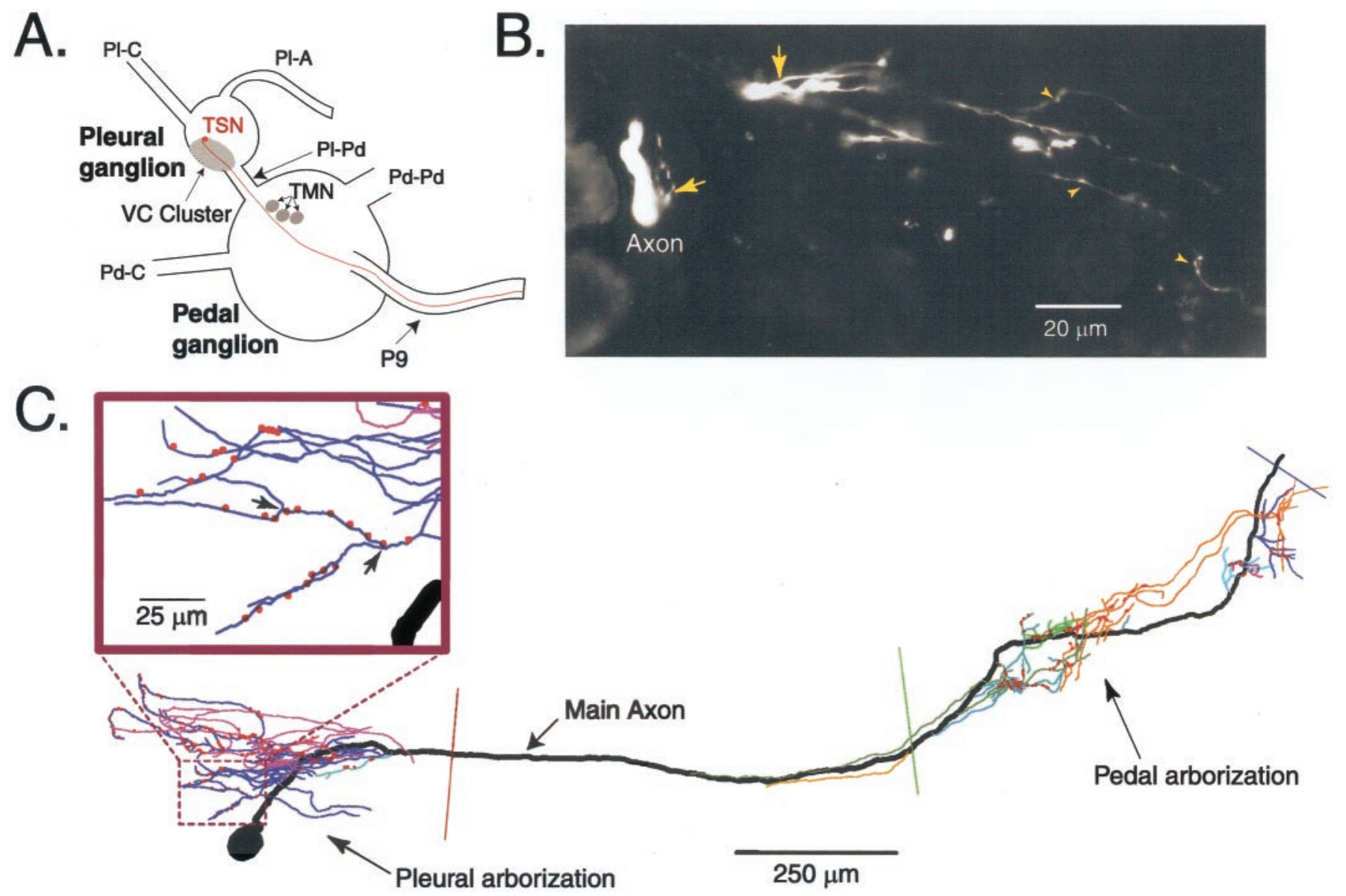

Figure 2. Reconstruction of tail sensory neurons. $A$, Schematic representation of tail sensory neurons in the CNS of Aplysia. The somata of tail sensory neurons $(T S N, r e d)$ lie in a cluster $(V C$ Cluster $)$ in the pleural ganglion. Sensory neurons project through the pleural-pedal connective $(P l-P d)$ into the pedal ganglion, where they make monosynaptic contacts onto one or more tail motor neurons (TMN) (Walters et al., 1983a). From the pedal ganglion they extend to the tail through the peripheral nerve P9. The pleural abdominal $(P l-A)$, pleural-cerebral $(P l-C)$, and pedal-cerebral $(P d-C)$ connectives and the pedal-pedal commissure $(P d-P d)$ are shown for orientation. $B$, Photomicrograph of a $40 \mu \mathrm{m}$ section in the pleural ganglion. The main axon of the filled sensory neuron can been seen on the left-hand side. Branch points are indicated by arrows, and varicosities are indicated by arrowheads. The complete sensory neuron was reconstructed from serial sections. $C$, Representative example of a reconstructed tail sensory neuron. Lines perpendicular to the main axon indicate the boundary lines between the pleural ganglion and the connective (red), the connective and the pedal ganglion (green), and the pedal ganglion and P9 (blue). The different colored branches represent individual arborizations (i.e., first-order branches and their corresponding higher order processes) emerging from the main axon. Filled red circles indicate varicosities. Inset is magnified to illustrate branch points (arrows) and varicosities.

cells from ganglia ipsilateral and contralateral to the training site. Consequently, data were pooled into two groups for comparison of trained and untrained animals. Training was associated with a significant increase in the number of varicosities along processes of sensory neurons from trained animals, but none of the other parameters measured were significantly altered (Fig. $3 B$ ).

To examine the possibility that structural changes were localized, pleural and pedal arborizations were analyzed separately (Fig. 3C,D). There was no effect of sensitization training on sensory neuron structure in the pleural ganglion (Fig. 3D1), but robust changes were observed in the pedal ganglion (Fig. 3D2). In this region, the arborization exhibited nearly a doubling in length and in the number of branch points. The number of varicosities increased more than threefold compared with untrained controls. The number of first-order branches was not significantly different between the two groups.

\section{One day of training produced long-term sensitization that was not associated with neuronal outgrowth}

Animals exposed to sensitization training for $1 \mathrm{~d}$ also showed a unilateral enhancement of the tail-induced siphon withdrawal reflex $24 \mathrm{hr}$ after training [data from Cleary et al. (1998)] (Fig. $4 A)$. The reflex was enhanced on the side ipsilateral to the sensitizing stimulus $(182 \pm 12 \%$; mean \pm SEM $)$ but not on the contralateral side $\left(112 \pm 6 \% ; t_{82}=5.16 ; p<0.0001\right)$. This behavioral enhancement was correlated with lateralized changes in the membrane properties of the tail sensory and motor neurons and with the strength of the sensory to motor neuron connection (Cleary et al., 1998).

Tail sensory neurons from the same ganglia used for electrophysiological analysis were injected for morphological analysis. We found no differences comparing sensory neurons ipsilateral to the training site with those contralateral. As above, the arborizations from the pleural and pedal ganglia were also analyzed separately, but no differences between groups were uncovered (Fig. 4B,C). In this experiment, untrained animals were not used. Consequently, we could not rule out the possibility of a bilateral enhancement.

In a separate study, however, the cells from animals exposed to the $1 \mathrm{~d}$ training protocol were compared with those from untrained controls. In this experiment, the intertrain interval was 12 


\section{A. 4-day Training}



\section{B. Entire cell}

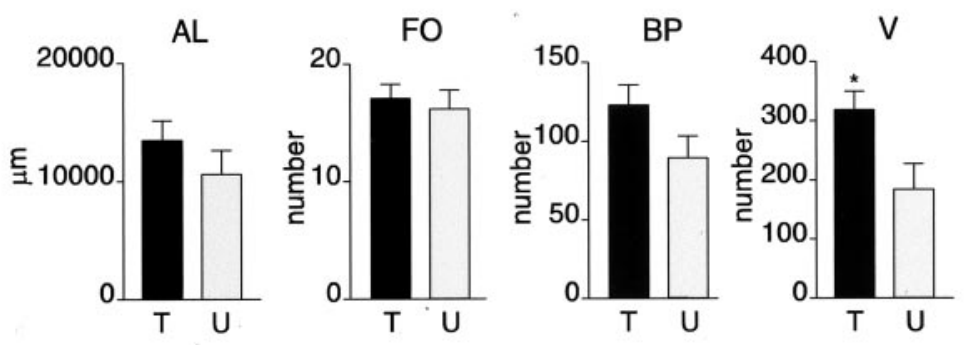

C1. Untrained
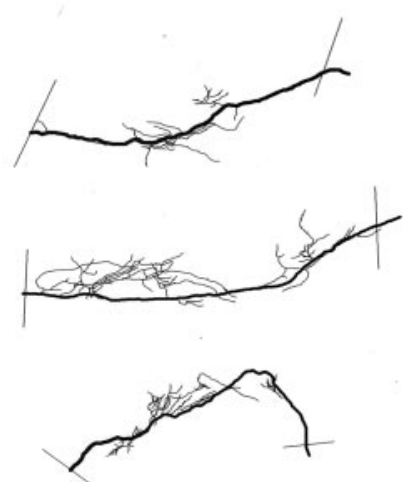

C2.



D1. Pleural Arborization
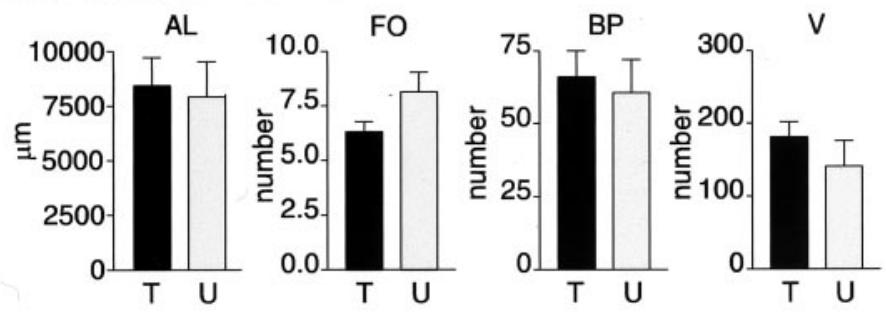

D2. Pedal Arborization


Figure 3. Four days of training induced structural changes in the pedal ganglion. $A$, Four days of sensitization training produced a robust sensitization of the tail-siphon withdrawal reflex. Twenty-four hours after the end of training, the duration of the siphon withdrawal in response to mild tail stimulation was significantly longer on the side of the animals ipsilateral to the sensitizing stimuli $(I, n=11)$ than on the contralateral side $(C, n=11)$ or in untrained control animals $(U, n=18)$. All values are expressed as the percentage increase of post-test values over pre-test values (one-way ANOVA with Tukey test for multiple comparisons; $\left.{ }^{*} p<0.001\right)$. $B$, The $4 \mathrm{~d}$ training protocol induced modest changes in overall sensory neuron morphology. There was an increase in the number of varicosities along sensory neuron processes in trained animals $(T$, ipsilateral and contralateral sides pooled; $n=16)$ compared with untrained controls $(U, n=7)$, but none of the other parameters measured were significantly altered by training (Student's $t$ test; $\left.{ }^{*} p<0.05\right)$. $A L$, Arborization length; $F O$, first-order branches; $B P$, branch points; $V$, varicosities. $C$, Examples of sensory neuron arborizations in the pedal ganglion from untrained $(C 1)$ and $4 \mathrm{~d}$ trained $(C 2)$ animals. Arborizations from trained animals appear more extensive than those from controls. Varicosities are not visible at this scale. The straight lines perpendicular to the main axon (thick process) represent the point at which the sensory neuron enters the pedal ganglion through the pleural-pedal connective (left) and the point at which it exits the pleural ganglion through the peripheral nerve, P9 (right). D, Group analysis of sensory neuron morphology from animals trained with the 4 day protocol revealed significant outgrowth in the pedal ganglion. There were no significant differences between the pleural arborizations $(D 1)$ of sensory neurons from trained $(T, n=16)$ and untrained $(U, n=7)$ animals for any of the parameters measured. However, in the pedal ganglion $(D 2)$, trained animals exhibited increased arborization length $(A L)$, number of branch points $(B P)$, and number of varicosities $(V)$, compared with untrained controls (Student's $t$ test; ${ }^{*} p<0.05$, **indicates $p<0.005$ ).

min. At 24 hr after training, tail-induced siphon withdrawal was significantly enhanced $\left(F_{(2,83)}=6.47 ; p<0.005\right)$. Sensitization occurred on the side ipsilateral to the training site compared with untrained controls $(200 \pm 33$ vs $111 \pm 7 \% ; q=4.78 ; p<$ 0.01 ; Tukey). The response on the contralateral side was not significantly enhanced compared with untrained animals (168 \pm 27 vs $111 \pm 7 \% ; q=3.1 ; p>0.05$; Tukey). The sensory neurons from the trained animals were identical to those from untrained animals, ruling out the possibility that bilateral changes in morphology had occurred (Table 1). These results indicate that $1 \mathrm{~d}$ training is not associated with neuronal outgrowth.
Just as $1 \mathrm{~d}$ of sensitization training was shown to produce a long-term enhancement of synaptic strength (Cleary et al., $1998)$, the growth factor TGF- $\beta$ has been shown to induce long-term facilitation in isolated pleural-pedal ganglia (Zhang et al., 1997). Application of TGF- $\beta$ induced facilitation of the sensorimotor connection lasting at least $24 \mathrm{hr}$ after the end of treatment. In this study, single sensory neurons from the same preparations used for electrophysiological analysis (Zhang et al., 1997) were injected with Rh-dextran and processed for morphological analysis. The long-term facilitation induced by TGF- $\beta$ was not associated with structural changes in the tail sensory neuron (data not shown). 
A. One-day Training

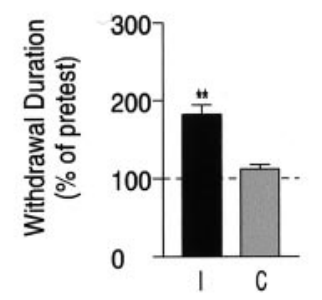

\section{B1. Contralateral}



C1. Pleural Arborization

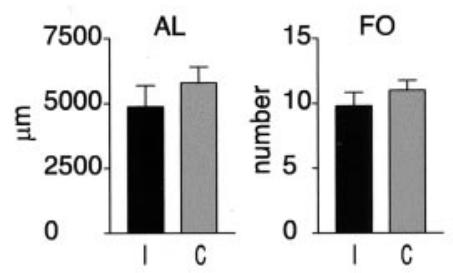

C2. Pedal Arborization


Figure 4. One day of sensitization training is not associated with neuronal outgrowth after $24 \mathrm{hr}$. A, One day of training produced unilateral sensitization of the tail-induced siphon withdrawal reflex. The duration of the siphon withdrawal in response to mild tail stimulation was significantly longer $24 \mathrm{hr}$ after training on the side of the animals receiving sensitizing stimuli $(I, n=42)$ than on the contralateral side $(C, n=42)$ (Student's $t$ test; $\left.{ }^{* *} p<0.0001\right)$. Data reproduced from Cleary et al. (1998). B, Examples of sensory neuron arborizations from Contralateral (B1) and Ipsilateral (B2) sides of $1 \mathrm{~d}$ trained animals. There appear to be no differences in complexity of arborizations between the two groups. Varicosities are not visible at this scale. The straight lines perpendicular to the main axon (thick process) represent the point at which the sensory neuron enters the pedal ganglion through the pleural-pedal connective (left) and the point at which it exits the pleural ganglion through the peripheral nerve, $\mathrm{P} 9$ (right). $C$, Group analysis of the morphology of sensory neurons from animals tested $24 \mathrm{hr}$ after training revealed no significant differences between sensory neurons in ganglia ipsilateral to the side of sensitization training $(I, n=15)$ and those from the side contralateral to training $(C$, $n=15)$ in either the pleural $(C 1)$ or pedal $(C 2)$ ganglion. Averages were compared using Student's two-tailed $t$ test. $A L$, Arborization length; $F O$, first-order branches; $B P$, branch points; $V$, varicosities.
Table 1. Sensory neuron structure as measured $24 \mathrm{hr}$ after $1 \mathrm{~d}$ training (trained vs untrained animals)

\begin{tabular}{|c|c|c|c|c|}
\hline \multirow[b]{2}{*}{ Parameter } & \multirow{2}{*}{$\begin{array}{l}\text { Trained } \\
(n=7)\end{array}$} & \multirow{2}{*}{$\begin{array}{l}\text { Untrained } \\
(n=7)\end{array}$} & \multicolumn{2}{|c|}{$\begin{array}{l}\text { Statistical } \\
\text { values }\end{array}$} \\
\hline & & & $t_{21}$ & $p$ \\
\hline \multicolumn{5}{|l|}{ Entire cell } \\
\hline Arborization length $(\mu \mathrm{m})$ & $9652 \pm 1617$ & $9593 \pm 1146$ & 0.18 & 0.86 \\
\hline \multicolumn{5}{|l|}{ Number of first-order } \\
\hline branches & $20.6 \pm 1.9$ & $24.3 \pm 4.4$ & 0.78 & 0.45 \\
\hline Number of branch points & $121.4 \pm 23.3$ & $113.1 \pm 15.9$ & 0.29 & 0.77 \\
\hline Number of varicosities & $220.3 \pm 44.9$ & $281.7 \pm 57.4$ & 0.84 & 0.42 \\
\hline \multicolumn{5}{|l|}{ Pleural arborization } \\
\hline Arborization length & $6068 \pm 862$ & $6239 \pm 802$ & 0.15 & 0.89 \\
\hline \multicolumn{5}{|l|}{ Number of first-order } \\
\hline branches & $10.3 \pm 1.6$ & $12.3 \pm 2.8$ & 0.62 & 0.55 \\
\hline Number of branch points & $72.0 \pm 13.5$ & $73.6 \pm 10.1$ & 0.09 & 0.93 \\
\hline Number of varicosities & $142.3 \pm 25.3$ & $201.43 \pm 49.5$ & 1.06 & 0.31 \\
\hline \multicolumn{5}{|l|}{ Pedal arborization } \\
\hline Arborization length & $3574 \pm 719$ & $3296 \pm 715$ & 0.27 & 0.79 \\
\hline \multicolumn{5}{|l|}{ Number of first-order } \\
\hline branches & $10.7 \pm 0.84$ & $12.3 \pm 2.0$ & 0.71 & 0.49 \\
\hline Number of branch points & $48.6 \pm 10.3$ & $41.0 \pm 9.9$ & 0.53 & 0.60 \\
\hline Number of varicosities & $78.3 \pm 21.9$ & $80.3 \pm 23.1$ & 0.06 & 0.95 \\
\hline
\end{tabular}

All values expressed as mean \pm SEM. Entire cell refers to the analysis of the sensory neuron structure throughout the pleural and pedal ganglia. Pleural and pedal values refer to separate analysis of the pleural and pedal arborization of the same population of cells. Trained, Cells from ipsilateral and contralateral sides of trained animals; Untrained, cells from untrained controls. $p$ values determined by Student's unpaired two-tailed $t$ test.

\section{Delayed induction of neurite outgrowth was not observed after $1 \mathrm{~d}$ training}

It is possible that outgrowth was induced by the $1 \mathrm{~d}$ training protocol but not observed because the latency for expression was $>24 \mathrm{hr}$. To explore this possibility, we examined sensory neuron morphology $4 \mathrm{~d}$ after $1 \mathrm{~d}$ sensitization training (the same time point, from the start of training, that neurons from the $4 \mathrm{~d}$ trained animals were examined). Animals were exposed to the $1 \mathrm{~d}$ protocol, and behavioral testing was conducted at $24 \mathrm{hr}$ to test the strength of sensitization and at $4 \mathrm{~d}$ to test retention (Fig. $5 A$ ). The pleural-pedal ganglia were removed immediately after the second post-test for morphological analysis.

Behaviorally, we found that $1 \mathrm{~d}$ of training induced a unilateral long-term sensitization that lasted $>24 \mathrm{hr}$ but $<4 \mathrm{~d}$ (Fig. 5B). Twenty-four hours after training (Fig. 5B1), siphon withdrawal duration was significantly enhanced $\left(F_{(2,35)}=9.3 ; p<0.001\right)$. Withdrawal duration was significantly greater in response to mild tail stimulation on the side ipsilateral to the sensitization training $(I, 149 \pm 18 \%$; mean \pm SEM) than on the contralateral side $(C$, $82 \pm 9 \% ; q=5.43 ; p<0.01$; Tukey) or in untrained control animals $(U, 82 \pm 7 \% ; q=4.95 ; p<0.01)$. By $4 \mathrm{~d}$ after training (Fig. 5B2), there were no significant differences among groups (I, $\left.119 \pm 11 \% ; C, 108 \pm 10 \% ; U, 105 \pm 8 \% ; F_{(2,35)}=0.50 ; p=0.61\right)$, indicating that the memory for sensitization had decayed by this time. This is similar to the duration of sensitization produced by $1 \mathrm{~d}$ of training in the siphon-gill withdrawal reflex (Frost et al., 1985).

Morphological analysis of sensory neurons $4 \mathrm{~d}$ after $1 \mathrm{~d}$ sensitization training revealed no significant differences between neurons from trained and untrained animals. Sensory neuron arborizations in the pleural and pedal ganglia were analyzed 
A. 1-day Training

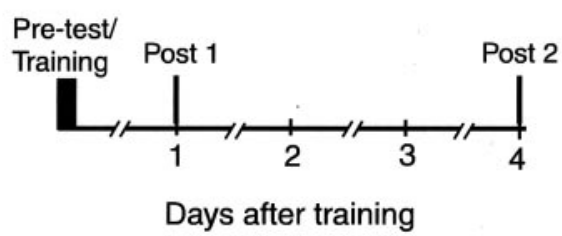

B1. 24 Hour Post-test

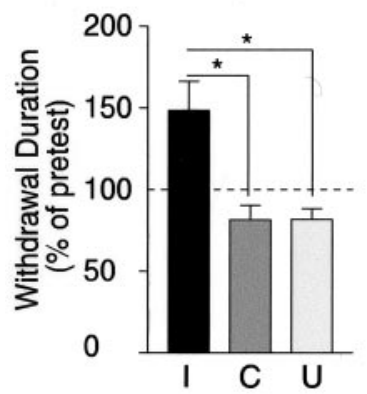

B2. 4 Day Post-test

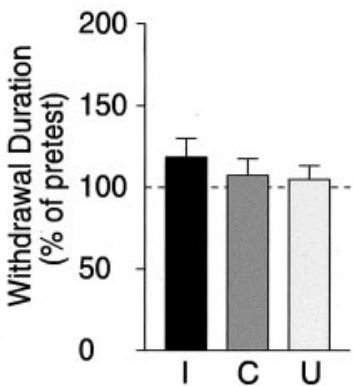

\section{C1. Pleural Arborization}
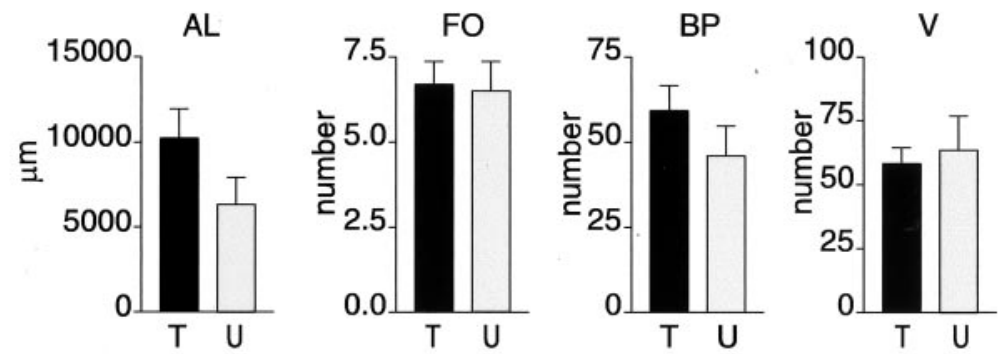

\section{C2. Pedal Arborization}
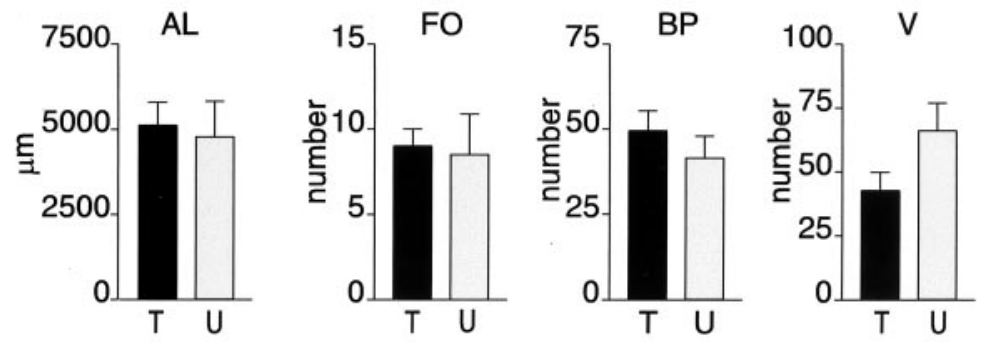

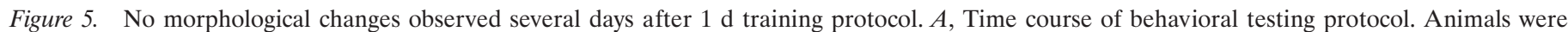

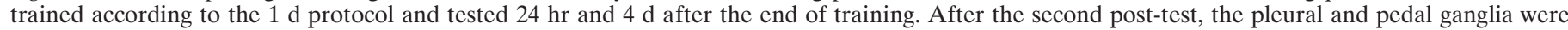

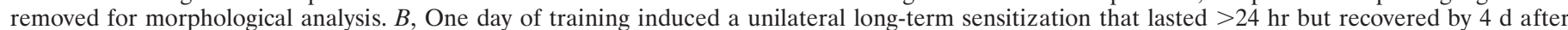

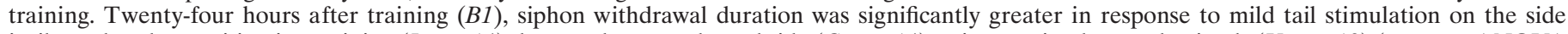



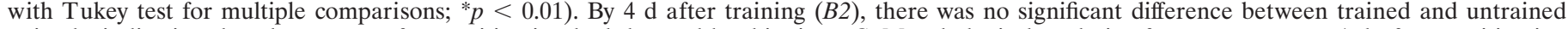



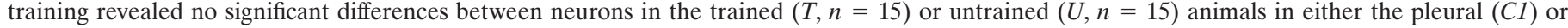


points; $V$, varicosities.

separately to detect subtle or localized changes with the same results (Fig. 5C1,C2). The fact that sensory neuron structure remained stable $4 \mathrm{~d}$ after training excludes the possibility that $1 \mathrm{~d}$ of sensitization training induced morphological outgrowth with a slow onset of expression and confirmed that this protocol was not sufficient to induce neurite outgrowth in tail sensory neurons.

\section{Massed training was ineffective in inducing long-term sensitization}

Another possible explanation for the difference between the two training protocols was that the $1 \mathrm{~d}$ protocol simply consisted of fewer training trials and was therefore not sufficient to produce sensitization comparable with that induced by the $4 \mathrm{~d}$ protocol. We examined this possibility by extending the $1 \mathrm{~d}$ protocol to consist of the same number of stimuli as the $4 \mathrm{~d}$ protocol (i.e., 16 rather than 4 trains of stimuli were administered at $30 \mathrm{~min}$ intervals) (Fig. 1D).

Surprisingly, we found that animals exposed to this massed protocol showed no sensitization $24 \mathrm{hr}$ after training (Fig. 6A). Although there was a trend toward greater siphon withdrawal duration after training on the side ipsilateral to the stimulation compared with the contralateral side, neither side was different from untrained controls $(I, 91 \pm 38 \%, n=9 ; C, 77 \pm 8 \%, n=9$; $U, 103.1 \pm 13 \%, n=11 ;$ mean $\left.\pm \operatorname{SEM} ; F_{(2,26)}=1.33 ; p=0.28\right)$.

Despite the relatively intense stimulation, there were no differences in morphology between the sensory neurons from trained and untrained animals (Fig. 6B1,B2). Moreover, the lack of structural changes suggests that the outgrowth induced by the $4 \mathrm{~d}$ training protocol is not a function of the amount of stimulation but appears to be dependent on the temporal spacing of the stimulation over multiple days.

\section{DISCUSSION}

Neuronal outgrowth is a correlate of long-term sensitization after extended training

Four days of sensitization training induced a robust long-term sensitization, confirming that $4 \mathrm{~d}$ training effectively induces longterm sensitization in the tail-induced tail-siphon withdrawal reflex. This protocol also produced changes in the morphology of tail sensory neurons comparable to those observed in siphon sensory neurons (Bailey and Chen, 1988a). However, in contrast to previous studies in siphon sensory neurons, only a subset of the tail sensory neuron arborizations appeared to be affected. The restriction of outgrowth to the region in closest proximity to the follower motor neurons is consistent with the possibility that outgrowth contributes to the enhancement of the tail withdrawal component of the reflex after sensitization training (but see next section).

The localization of outgrowth to the pedal ganglion is a provocative result. Because sensitizing stimuli do not directly activate tail sensory neurons, one might expect a diffuse modulatory pathway to be involved. For example, serotonergic pathways have been implicated in sensitization (Glanzman et al., 1989), and serotonin-immunoreactive neurites permeate the entire CNS. Consequently, we initially expected the entire arborization of the sensory neuron to be affected. Functionally, this would be appropriate because there are follower neurons (excitatory and inhib- 


\section{A. Massed Training}

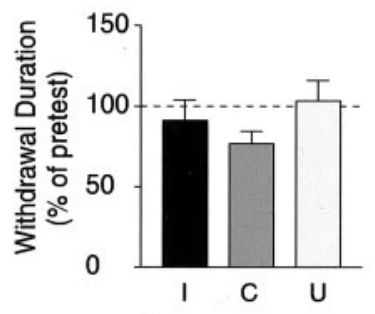

B1. Pleural Arborization


B2. Pedal Arborization


Figure 6. Massed training does not induce long-term sensitization or structural changes in tail sensory neurons. $A$, Twenty-four hours after training, siphon withdrawal duration was not significantly different in response to mild tail stimulation on the side ipsilateral to the sensitization training $(I, n=9)$ than on the contralateral side $(C, n=9)$ or in untrained control animals $(U, n=11)$. Averages were compared using a one-way ANOVA. B, Morphological analysis of tail sensory neurons $24 \mathrm{hr}$ after $1 \mathrm{~d}$ of massed training revealed no significant differences between neurons in the ganglia from trained $(T, n=10)$ and untrained $(U, n=6)$ animals in either the pleural (B1) or pedal (B2) ganglion. Averages were compared using Student's unpaired two-tailed $t$ test. $A L$, Arborization length; $F O$, first-order branches; $B P$, branch points; $V$, varicosities.

itory), the cell bodies of which are located in the pleural ganglion (Buonomano et al., 1992; Cleary and Byrne, 1993). At this point, we do not understand why the pleural arborization is unaffected. However, we cannot rule out the possibility that outgrowth is induced by training in some neurites but balanced by retraction in others, leading to no observable net change in that region.

The localized outgrowth suggests that cellular mechanisms exist that are capable of regulating outgrowth in a specific and relatively small fraction of the neuronal arborization. For example, one possible mechanism is the activation of local protein synthesis, which has been observed in neurites of cultured sensory neurons (Martin et al., 1997). Moreover, the localization of neurite outgrowth seems to be specific to sensitization. Tail sensory neurons can also undergo robust morphological changes after axonal injury, but this response is cell-wide and qualitatively distinct from the outgrowth observed after sensitization training (Wainwright et al., 1999).

In contrast to $4 \mathrm{~d}$ of training, $1 \mathrm{~d}$ of training was not sufficient to induce outgrowth in sensory neurons. This result was unexpected because the training was sufficient to induce a long-term behavioral change as well as several biophysical modifications in sensory neurons, including increased excitability, enhanced synaptic strength, and a reduction of net outward currents (Scholz and Byrne, 1987; Cleary et al., 1998). Moreover, intracellular injection of cAMP, a second messenger activated in sensory neurons by sensitizing stimuli (Ocorr et al., 1986), produced neurite outgrowth within 24 hr (Nazif et al., 1991; O'Leary et al., 1995).

It is important to emphasize that the current study focused only on neuronal outgrowth and varicosity formation, which might result in the formation of new synapses. Ultrastructural changes that affect the strength of preexisting synapses, such as formation of new active zones or growth and enhancement of existing release sites, have also been correlated with sensitization training (Bailey and Chen, 1988a,b, 1989). Our studies do not exclude the possibility that 1 or $4 \mathrm{~d}$ of training alters the strength of individual synapses through structural modifications of preexisting synapses.

In isolation, the morphological changes that we observed are consistent with a model in which neurite outgrowth contributes to the formation of new synapses, presumably enhancing the strength of existing neural circuits. However, this outgrowth appears to be associated only with some forms of long-term sensitization - the most enduring forms. According to this model, structural changes would have a high threshold for induction and be more difficult to reverse. Consequently, procedures like the $1 \mathrm{~d}$ protocol, which recover relatively quickly, would not elicit neurite outgrowth. On the other hand, the $4 \mathrm{~d}$ training protocol presumably produces a memory that persists for at least 3 weeks (Frost et al., 1985; Bailey and Chen, 1989) and is sufficient to activate outgrowth.

Interestingly, massed training did not induce outgrowth, which implies that the induction of robust and persistent memory depends not merely on the number of stimuli presented but on the spacing of the training trials over multiple days. This would be consistent with previous studies suggesting that spaced training protocols are more effective than massed training protocols (Yin et al., 1994; Mauelshagen et al., 1998; Carew et al., 2001; Sutton et al., 2002). Presumably this reflects the interaction of stimuli with intracellular mechanisms that have a relatively long time course (Kogan et al., 1997; Smolen et al., 1998). In Aplysia, these might include the expression of certain structural proteins (Noel et al., 1993) or the activation of growth factors (Zhang et al., 1997).

Surprisingly, massed training was not simply less effective but failed to induce long-term sensitization at all. We do not believe that this was due to a problem with the sample of animals, because other animals from the same shipment that were trained at the same time did show long-term sensitization. Moreover, this did not appear to be a performance deficit caused by injury. Although the training was more intense than usual, no evidence of injury was observed at the training site, and trained animals could not be distinguished from untrained on the basis of their ambient behavior. It is more likely that repeated training sessions activated inhibitory processes that blocked the expected enhancement. Short-term sensitization training activates inhibitory processes (Mackey et al., 1987; Marcus et al., 1988; Wright et al., 1991; Hawkins et al., 1998). These changes are too transient to affect the enhancement seen after $24 \mathrm{hr}$, but perhaps long-term enhancement of these processes or induction of additional inhibitory processes (Pettigrew et al., 2001) occurs with a higher threshold. Interestingly, massing the stimuli presented in a $1 \mathrm{~d}$ protocol (e.g., four to five shocks) by decreasing the interstimulus interval induces intermediate but not long-term sensitization 
(Carew et al., 2001, Sutton et al., 2002), which provides further support for the notion that the temporal pattern of training stimuli is key to the induction of lasting memories.

\section{Causal role of neuronal outgrowth in long-term sensitization}

These results clearly demonstrate that $4 \mathrm{~d}$ of sensitization training are associated with outgrowth of pleural sensory neurons. Nevertheless, the current study raises some important questions about the role of these changes in long-term sensitization. In this study we observed two major dissociations between changes in sensory neuron morphology and enhancement of the siphon withdrawal response. First, with the $1 \mathrm{~d}$ training protocol, we were unable to observe large-scale morphological changes, although the behavior was reliably enhanced. Neurite outgrowth was not expressed even when given 3 additional days. Biophysical changes at the cellular level have been observed after this protocol, and these changes occur on the trained side of the animal (Scholz and Byrne, 1987; Cleary et al., 1998). Moreover, application of TGF- $\beta$, an in vitro analog of this procedure, induced long-term facilitation in the absence of observable outgrowth. These observations support the idea that this form of long-term sensitization is caused by modification of preexisting synapses rather than formation of new synapses.

The second dissociation that we observed followed $4 \mathrm{~d}$ of training, when morphological changes on the contralateral side of the animal occurred in the absence of a behavioral modification. However, this finding can be interpreted in several ways. One possible explanation is that structural changes in sensory neurons are not by themselves sufficient to affect the reflex. Morphological changes could be necessary, however, if there are other circuit modifications downstream that are lateralized. For example, new branches may form competent synapses only on the sensitized side of the animal. We cannot rule out the possibility, however, that the tail withdrawal component of the reflex, unlike the siphon withdrawal component, is indeed sensitized bilaterally.

Nevertheless, bilateral outgrowth may force us to reexamine the assumption that the tail and siphon components of the response are modulated identically. This assumption has never been proven because of the difficulty of accurately measuring the tail withdrawal component of the reflex. As a first step, future experiments will examine the biophysical correlates of the $4 \mathrm{~d}$ training protocol to determine whether contralateral outgrowth is correlated with enhanced synaptic strength.

Despite much effort, it has been difficult to prove that changes in morphology produce changes in behavior. Morphological changes have consistently been correlated with long-term sensitization in Aplysia, yet we have observed dissociations between these changes and the modification of the behavior that cast doubt on their causal relationship. Similarly, recent evidence from cultured Aplysia sensory-motor neurons suggests that some forms of long-term facilitation are not associated with increased numbers of presynaptic varicosities (Casadio et al., 1999). In the hippocampus, there are many examples of correlations between long-term potentiation and changes in the morphology of dendrites, yet there is still no definitive evidence that these morphological changes are functionally relevant (for review, see Agnihotri et al., 1998; Yuste and Bonhoeffer, 2001). Moreover, studies at the Drosophila neuromuscular junction have shown that an alteration in the number of presynaptic boutons does not necessarily translate into altered synaptic efficacy (Stewart et al., 1996), suggesting that changes in morphology may only provide a framework within which mechanisms of plasticity work. These studies, along with the present study, suggest that although gross morphological changes might play a role in long-term synaptic plasticity, the relationship between structure and function may not be as simple as was once thought.

\section{REFERENCES}

Advokat C (1980) Modulation of defensive reflexes in Aplysia californica by appetitive stimulation. Behav Neural Biol 28:253-265.

Agnihotri N, Lopez-Garcia JC, Hawkins RD, Arancio O (1998) Morphological changes associated with long-term potentiation. Histol Histopathol 13:1155-1162.

Bailey CH, Chen M (1983) Morphological basis of long-term habituation and sensitization in Aplysia. Science 220:91-93.

Bailey CH, Chen M (1988a) Long-term memory in Aplysia modulates the total number of varicosities of single identified sensory neurons. Proc Natl Acad Sci USA 85:2373-2377.

Bailey CH, Chen M (1988b) Long-term sensitization in Aplysia increases the number of presynaptic contacts onto the identified gill motor neuron L7. Proc Natl Acad Sci USA 85:9356-9359.

Bailey CH, Chen M (1989) Time course of structural changes at identified sensory neuron synapses during long-term sensitization in Aplysia. J Neurosci 9:1774-1780.

Bailey CH, Kandel ER (1993) Structural changes accompanying memory storage. Annu Rev Physiol 55:397-426.

Bailey CH, Thompson EB, Castellucci VF, Kandel ER (1979) Ultrastructure of the synapses of sensory neurons that mediate the gillwithdrawal reflex in Aplysia. J Neurocytol 8:415-444.

Buonomano DV, Cleary LJ, Byrne JH (1992) Inhibitory neuron produces heterosynaptic inhibition of the sensory-to-motor neuron synapse in Aplysia. Brain Res 577:147-150.

Byrne JH, Castellucci VF, Kandel ER (1974) Receptive fields and response properties of mechanoreceptor neurons innervating siphon skin and mantle shelf of Aplysia. J Neurophysiol 37:1041-1064.

Carew TJ, Castellucci VF, Kandel ER (1971) An analysis of dishabituation and sensitization of the gill-withdrawal reflex in Aplysia. Int J Neurosci 2:79-98.

Carew TJ, Ide J, Masters SE, Sutton MA (2001) Amount and pattern of training in the induction of intermediate- and long-term memory in Aplysia. Soc Neurosci Abstr 27:2534.

Casadio A, Martin KC, Giustetto M, Z hu H, Chen M, Bartsch D, Bailey CH, Kandel ER (1999) A transient, neuron-wide form of CREBmediated long-term facilitation can be stabilized at specific synapses by local protein synthesis. Cell 992:221-237.

Castellucci VF, Pinsker H, Kupfermann I, Kandel ER (1970) Neuronal mechanisms of habituation and dishabituation of the gill withdrawal reflex in Aplysia. Science 167:1745-1748.

Castellucci VF, Blumenfeld H, Goelet P, Kandel ER (1989) Inhibitor of protein synthesis blocks long-term behavioral sensitization in the isolated gill-withdrawal reflex of Aplysia. J Neurobiol 20:1-19.

Cleary LJ, Byrne JH (1993) Identification and characterization of a multif unction interneuron contributing to defensive arousal in Aplysia. J Neurophysiol 70:1767-1776.

Cleary LJ, Byrne JH, Frost WN (1995) Role of interneurons in defensive withdrawal reflexes in Aplysia. Learn Mem 2:133-151.

Cleary LJ, Lee WL, Byrne JH (1998) Cellular correlates of long-term sensitization in Aplysia. J Neurosci 18:5988-5998.

Domjan M (1993) The principles of learning and memory, Ed 3. Pacific Grove, CA: Brooks/Cole.

Dudai Y (1989) The neurobiology of memory: concepts, findings, trends. New York: Oxford UP.

Frost WN, Castellucci VF, Hawkins RD, Kandel ER (1985) Monosynaptic connections made by the sensory neurons of the gill- and siphonwithdrawal reflex in Aplysia participate in the storage of long-term memory for sensitization. Proc Natl Acad Sci USA 82:8266-8269.

Glanzman DL, Mackey SL, Hawkins RD, Dyke AM, Lloyd PE, Kandel ER (1989) Depletion of serotonin in the nervous system of Aplysia reduces the behavioral enhancement of gill withdrawal as well as the heterosynaptic facilitation produced by tail shock. J Neurosci 9:4200-4213

Glanzman DL, Kandel ER, Schacher S (1990) Target-dependent structural changes accompanying long-term synaptic facilitation in Aplysia neurons. Science 249:799-802.

Goldsmith JR, Byrne JH (1993) Bag cell extract inhibits tail-siphon withdrawal reflex, suppresses long-term but not short-term sensitization, and attenuates sensory-to-motor synapses in Aplysia. J Neurosci 13:1688-1700.

Greenough WT, Bailey CH (1988) The anatomy of a memory: convergence of results across a diversity of tests. Trends Neurosci 11:142-147.

Hawkins RD, Cohen TE, Greene W, Kandel ER (1998) Relationships between dishabituation, sensitization, and inhibition of the gill- and 
siphon-withdrawal reflex in Aplysia californica: effects of response measure, test time, and training stimulus. Behav Neurosci 112:24-38.

Kogan JH, Frankland PW, Blendy JA, Coblentz J, Marowitz Z, Schutz G, Silva AJ (1997) Spaced training induced normal long-term memory in CREB mutant mice. Curr Biol 7:1-11.

Levenson J, Byrne JH, Eskin A (1999) Levels of serotonin in the hemolymph of Aplysia are modulated by light/dark cycles and sensitization training. J Neurosci 19:8094-8103.

Mackey SL, Glanzman DL, Small SA, Dyke AM, Kandel ER, Hawkins RD (1987) Tail shock produces inhibition as well as sensitization of the siphon-withdrawal reflex of Aplysia: possible behavioral role for presynaptic inhibition mediated by the peptide Phe-Met-Arg-Phe-NH2. Proc Natl Acad Sci USA 84:8730-8734.

Marcus EA, Nolen TG, Rankin CH, Carew TJ (1988) Behavioral dissociation of dishabituation, sensitization, and inhibition in Aplysia. Science 241:210-213.

Martin KC, Casadio A, Zhu H, Yaping E, Rose JC, Chen M, Bailey CH, Kandel ER (1997) Synapse-specific, long-term facilitation of Aplysia sensory to motor synapses: a function for local protein synthesis in memory storage. Cell 91:927-938.

Mauelshagen J, Sherff C, Carew TJ (1998) Differential induction of long-term synaptic facilitation by spaced and massed applications of serotonin at sensory neuron synapses of Aplysia californica. Learn Mem 5:246-256.

Montarolo PG, Goelet P, Castellucci VF, Morgan J, Kandel ER, Schacher SA (1986) A critical period for macromolecular synthesis in long-term heterosynaptic facilitation in Aplysia. Science 234:1249-1254.

Nazif F, Byrne JH, Cleary LJ (1991) cAMP induces long-term morphological changes in sensory neurons of Aplysia. Brain Res 539:324-327.

Noel F, Nuñez-Regueiro M, Cook R, Byrne JH, Eskin A (1993) Longterm changes in synthesis of intermediate filament protein, actin and other proteins in pleural sensory neurons of Aplysia produced by an in vitro analogue of sensitization training. Brain Res 19:203-210.

Ocorr KA, Tabata M, Byrne JH (1986) Stimuli that produce sensitization lead to elevation of cyclic AMP levels in tail sensory neurons of Aplysia. Brain Res 371:190-192.

O'Leary FA, Byrne JH, Cleary LJ (1995) Long-term structural remodeling in Aplysia sensory neurons requires de novo protein synthesis during a critical time period. J Neurosci 15:3519-3525.

Perlman AJ (1979) Central and peripheral control of siphon-withdrawal reflex in Aplysia californica. J Neruophysiol 42:510-529.

Pettigrew DB, Smolen P, Baxter DA, Byrne JH (2001) Spaced, but not massed, stimulation induces long-term facilitation in an intracellular model of Aplysia sensory neurons. Soc Neurosci Abstr 27:2535.

Pinsker HM, Hening WA, Carew TJ, Kandel ER (1973) Long-term sensitization of a defensive withdrawal reflex in Aplysia. Science 182:1039-1042.

Ramón y Cajal S (1988) Anatomicophysiological considerations on the cerebrum. In: Cajal on the cerebral cortex: an annotated translation of the complete writings. (DeFelipe J, Jones EG, eds), pp 465-490, New York: Oxford UP.

Rosenzweig MR (1996) Aspects of the search for neural mechanisms of memory. Annu Rev Psychol 47:1-32.

Schacher S, Kandel ER, Montarolo P (1993) cAMP and arachidonic acid simulate long-term structural and functional changes produced by neurotransmitters in Aplysia sensory neurons. Neuron 10:1079-1088.

Scholz KP, Byrne JH (1987) Long-term sensitization in Aplysia: biophysical correlates in tail sensory neurons. Science 235:685-687.

Smolen P, Baxter DA, Byrne JH (1998) Frequency selectivity, multistability, and oscillations emerge from models of genetic regulatory systems. Am J Physiol 274:C531-542.

Stewart BA, Schuster CM, Goodman CS, Atwood HL (1996) Homeostasis of synaptic transmission in Drosophila with genetically altered nerve terminal morphology. J Neurosci 16:3877-3886.

Sutton MA, Ide J, Masters SE, Carew TJ (2002) Interaction between amount and pattern of training in the induction of intermediate- and long-term memory for sensitization in Aplysia. Learn Mem 9:29-40.

Wainwright ML, Zhang H, Byrne JH, Cleary LJ (1999) Structural plasticity of tail sensory neurons in Aplysia. Soc Neurosci Abstr 25:1611.

Walters ET (1987) Multiple sensory neuron correlates of site-specific sensitization in Aplysia. J Neurosci 7:408-417.

Walters ET, Byrne JH, Carew TJ, Kandel ER (1983a) Mechanoafferent neurons innervating tail of Aplysia. I. Response properties and synaptic connections. J Neurophysiol 50:1522-1542.

Walters ET, Byrne JH, Carew TJ, Kandel ER (1983b) Mechanoafferent neurons innervating tail of Aplysia. II. Modulation by sensitizing stimuli. J Neurophysiol 50:1543-1559.

Wright WG, Marcus EA, Carew TJ (1991) A cellular analysis of inhibition in the siphon withdrawal reflex of Aplysia. J Neurosci 11:2498-2509.

Yin JCP, Wallach JS, Del Vecchio M, Wilder EL, Zhou H, Quinn WG, Tully T (1994) Induction of a dominant negative CREB transgene specifically blocks long-term memory in Drosophila. Cell 79:49-58.

Yuste R, Bonhoeffer T (2001) Morphological changes in dendritic spines associated with long-term synaptic plasticity. Annu Rev Neurosci 24:1071-1089.

Zhang F, Endo S, Cleary LJ, Eskin A, Byrne JH (1997) Role of transforming growth factor- $\beta$ in long-term synaptic facilitation in Aplysia. Science 275:1318-1320. 